\title{
Business Models and Ecosystem for Big Data
}

\author{
Sonja Zillner
}

\begin{abstract}
With the recent technical advances in digitalisation and big data, the real and the virtual worlds are continuously merging, which, again, leads to entire valueadded chains being digitalised and integrated. The increase in industrial data combined with big data technologies triggers a wide range of new technical applications with new forms of value propositions that shift the logic of how business is done. To capture these new types of value, data-driven solutions for the industry will require new business models. The design of data-driven AI-based business models needs to incorporate various perspectives ranging from customer and user needs and their willingness to pay for new data-driven solutions to data access and the optimal use of technologies, while taking into account the currently established relationships with customers and partners. Successful data-driven business models are often based on strategic partnerships, with two or more players establishing the basis for sustainable win-win situations through transparent resource-, investment-, risk-, data- and valuesharing. This chapter will explore the different data-driven business approaches and highlight in this context the importance of functioning ecosystems on the various levels. The chapter will conclude with an introduction to the data-driven innovation framework, a proven methodology to guide the systematic investigation of datadriven business opportunities while incorporating the dynamics of the underlying ecosystems.
\end{abstract}

Keywords Big data $\cdot$ Business models · Data-driven innovation - Data ecosystems · Data economy $\cdot$ Innovation ecosystem

\footnotetext{
S. Zillner $(\bowtie)$

Siemens AG, Munich, Germany

e-mail: sonja.zillner@siemens.com

(C) The Author(s) 2021

E. Curry et al. (eds.), The Elements of Big Data Value, https://doi.org/10.1007/978-3-030-68176-0_11
} 


\section{Introduction}

With the recent technical advances in digitalisation and big data, the real and the virtual worlds are continuously merging, which, again, leads to entire value-added chains being digitalised and integrated. For instance, in the manufacturing domain, all the way from the product design to on-site customer services, the entire valueadded chain is digitalised. The increase in industrial data combined with big data technologies triggers a wide range of new technical applications with new forms of value propositions that shift the logic of how business is done.

Big data brings new value to existing and new businesses (Zillner et al. 2017). It enables the optimisation of established internal processes, such as the optimisation of logistics and operations, as well as the basis to monetise new offerings. In general, four different areas of value creation and business models can be distinguished. First, the optimisation and improvement of existing businesses mainly relies on the analysis of available data sources. Second, the upgrading and revaluation of businesses mostly relies on the integration of additional (often external) data sources. Third, monetising describes the realisation of new business opportunities that make use of available data sources. Finally, breakthrough business encompasses new ventures that rely on new data sources, which are often realised with new partners or even within new value networks.

To capture these new types of value, data-driven solutions for the industry will require new business models. The design of data-driven AI-based business models needs to incorporate various perspectives ranging from customer and user needs, their willingness to pay for new data-driven solutions to data access and the optimal use of technologies while taking into account the currently established relationships with customers and partners. In other words, the definition of promising data-driven business opportunities requires balancing the technical aspects on the supply side and the user perspective and market dynamics on the demand side.

In addition, successful data-driven business models are often based on strategic partnerships with two or more players establishing the basis for sustainable win-win situations through transparent resource-, investment-, risk-, data- and value-sharing. To connect all the partners and stakeholders, functioning ecosystems for data sharing, innovation and building value chains are needed.

In this chapter we describe the aforementioned challenges in further detail. To address these challenges, we sketch how the data-driven innovation (DDI) framework can be used to scope data-driven business opportunities by leveraging all needed partners and stakeholders, as well as by continuously aligning the needs on the demand side and the capabilities on the supply side.

This chapter starts by detailing central big data business approaches complemented by some analysis and examples.

In what follows, Sect. 2 gives insights into the different big data business approaches complemented by industrial usage stories. Section 3 elaborates on the nature of data-driven business opportunities, while Sect. 4 highlights the importance of and different levels of data ecosystems. Section 5 gives a short introduction to the 


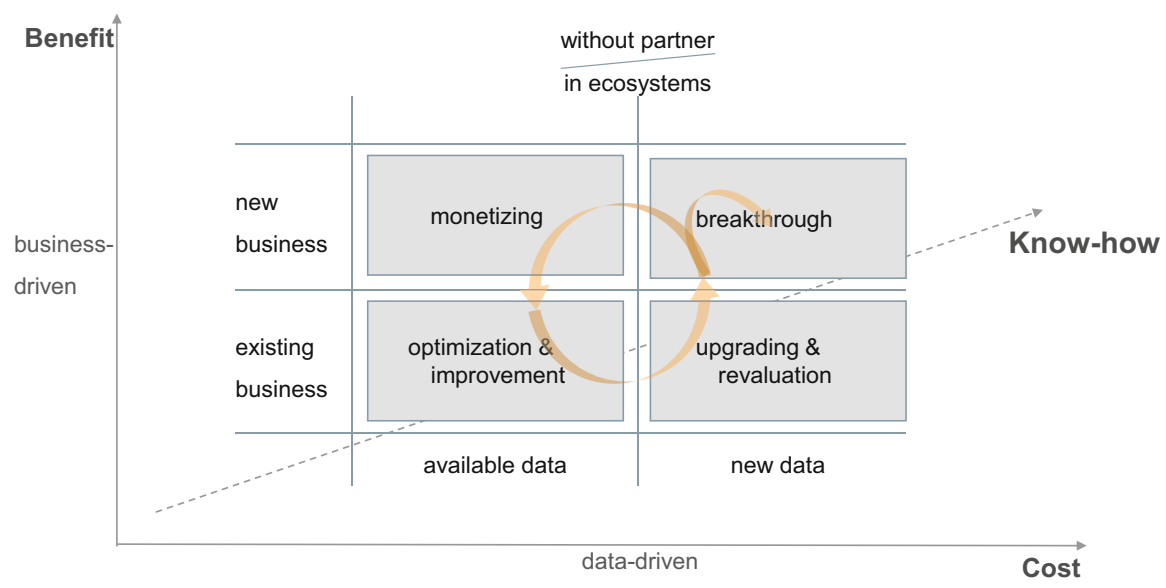

Fig. 1 Four variants of business patterns in the data economy (inspired by BITKOM 2013)

data-driven innovation framework as a possible way forward to scope data-driven business opportunities as well as adjacent ecosystems in a systematic manner. Section 6 concludes the chapter.

\section{Big Data Business Approaches}

The role of business models is to capture value from advancing technologies, such as big data. Business model decisions should not be driven by economic calculations only but should also consider the value opportunities for building up data asset and technology capability, as well as supporting ecosystems.

Within the data economy, we find various approaches to generating business value with big data technologies. ${ }^{1}$ Four generic business patterns (see Fig. 1) can be distinguished: One can generate business value by using existing data sources or by integrating additional and new data sources. The offerings can be realised by a single organisation or within an ecosystem of partners. In addition, the added value might help to improve existing products and services within an established market or can even be used to generate new businesses and sometimes even new markets.

In the following, we elaborate these four patterns by highlighting the involved costs as well as benefits. To capture the cost of each business pattern, we analyse the underlying data complexity and business complexity, as these two factors will

\footnotetext{
${ }^{1}$ Our findings are based on a series of expert interviews we accomplished with project leads/ participants of industrial big data projects.
} 
significantly drive the cost of implementation. To identify the benefits of each business pattern we refer to the value that is created. In addition, each business pattern will be illustrated with some industrial examples. We want to note that this simple classification clearly lacks scientific foundations. Its main objective is to provide strategic guidance for industrial decision makers when investing in big data projects.

\subsection{Optimisation and Improvements}

The business pattern "Optimisation and Improvements" relies on existing and already available data assets. These data assets require the typical efforts for data pre-processing and cleaning. Value is generated within the context of existing business processes.

Typical examples of this business pattern are as follows:

- Healthcare Domain: Administrative and financial data in hospital settings are analysed to increase the efficiency of the underlying administrative processes, such as scheduling of tasks or the utilisation of resources.

- Energy Domain: Sensor data of gas turbines are analysed to predict future damage and identify the cause of deviations in the process, in the material, etc.

For the above-mentioned examples as well as the business pattern in general, we can summarise the main characteristics of this business pattern.

Value Creation: The optimisation and improvement of existing process and businesses helps to reduce costs or to improve performance.

Data Complexity: In general, data assets are available, but their technical access needs to be ensured. Depending on the type of data source, e.g. sensor data or private data, the respective data governance challenges have to be addressed.

Business Complexity: The optimisation and improvement of established processes are in general a good starting point for data-enhanced offerings. By investigating available data sets, new insights regarding improvement potentials can be discovered while working with the data.

\subsection{Upgrading and Revaluation}

The business pattern "Upgrading and Revaluation" employs new data sources either by transforming internal raw data sources into a processable format (e.g. by semantic labelling of the content of medical images) or by integrating external data sources (e.g. weather forecast information) and developing new offerings.

Typical examples of this business pattern are as follows: 
- Energy Services Domain: Data- and knowledge-based services enable the discovery of new insights about trends that help to increase the overall business performance. Analytics applications are applied to all kinds of data (e.g. product data, market data, competitor data, web data, customer data, financial data) to detect and respond to product, event, personnel, competitor, customer and market trends.

- Healthcare Domain: A radiologist's workflow can be improved significantly by establishing the means for seamless navigation between medical image and dictated radiology report data. Content information of medical images as well as dictated medical reports is semantically described and linked by metadata.

- Industry Automation: The data from Large Hadron Collider (LHC) automation and control components and systems from Siemens (WINCC OA) are collected (offline and online) for automated system health check and diagnostics in order to prevent future damage, which helps to significantly reduce the overall maintenance cost.

- Global Production Chain: Intelligent integration of global supply chain management information (e.g. via object tracking information) into the production planning processes increases the robustness and efficiency of the global value chain.

- Smart Grid Systems: Optimised energy production through the interactive planning and optimising of the top-level design of microgrids in collaboration with the user. The interaction relies on visual result analysis that enables the user to detect patterns in large and heterogeneous data sets, such as weather data, power demand data, time series and energy capacity data.

For all of the above-mentioned examples as well as the business pattern in general, we can summarise the main characteristics of this big data business pattern.

Value Creation: Here the underlying idea is to upgrade existing business processes and services by making use of additional data sources. By aggregating multiple data sources, insights about process performances and operational and financial measures as well as guidance for business decisions can be provided.

Data Complexity: The integration of new data sources implies efforts and investments for handling the various data governance challenges. In addition to the challenge of accessing external data sources, one might face the challenge of overcoming internal data silos (sometimes even organisational silos), as well as the challenge of pre-processing raw data that is only available in an unstructured format, such as images, videos or dictated text.

Business Complexity: The described data governance challenges might imply high investments such that a long-term business strategy is needed. 


\subsection{Monetising}

The business pattern "Monetising" aims at generating new markets or revenue streams. By exploiting available data sources, completely new business scenarios, offerings and value streams are realised.

Typical examples of this big data business pattern are as follows:

- Clinical Research: Patient cohorts for clinical programmes can be identified more easily by using information extraction and advanced analytics on top of clinical data. In consequence, the feasibility of the clinical trials and thus the planning and designing of clinical programmes in the pharmaceutical domain can be significantly improved.

- Information Service: BLIDS ${ }^{2}$ is a lightning information service, which offers energy providers, industry, insurance companies or event organisers precise information about the number of registered lightning activities. The service aggregates weather data from approximately 8 countries and more than 145 measuring stations in Europe, as well as enabling the user-adapted representation of content.

- Smart Energy Profiles: Increasingly, metering service providers, which service renewable decentralised energy resources and new types of demand, such as electric vehicles, can bundle the characteristic information of power feed-in and energy usage into smart energy profiles and sell these for profit. Currently the whole energy market operates with standard load profiles, which are inefficient.

- Energy Automation: Intelligent electronic devices deliver real-time high-resolution data on power network parameters. When transmission network operators install these data sources near bigger renewable energy resources, and utilise advanced analytics, they can resell the information gained on the characteristics of the wind park and the network area back to the operator of the park for operational efficiency increase on both sides.

Value Creation: Secondary usage of data, i.e. the user benefiting from the collected data, is outside the original context in which the data were produced and collected.

Data Complexity: It is important here to clarify whether the usage of the available data sources for other purposes is legally allowed.

Business Complexity: Bringing data-driven offerings to the market might trigger the challenges of building new markets and establishing new or redefining established business relationships.

${ }^{2}$ Offered by Siemens. 


\subsection{Breakthrough}

Big data applications can lead to breakthrough scenarios that rely on collaborative ecosystems that establish new value networks by aggregating existing data sources with completely new data sources from various stakeholders.

Typical examples of the big data business pattern "Breakthrough" are as follows:

- Healthcare: Public health analytics application relies on the comprehensive disease management of chronic (e.g. diabetes, congestive heart failure) or severe (e.g. cancer) diseases that allow for the aggregation and analysis of treatment and outcome data, which again can be used to reduce complications, slow disease progression and improve outcomes.

- Energy Efficiency: Efficient energy use is highly dependent on energy automation - down to the device level in a private or commercial user. Learning systems are required which adapt to the preferences or business criteria of the energy user, along with efficient data exchange between retailers, energy markets and the network operators and the actual devices with the energy-efficiency service providers. Finally, smart meters and the metering service providers enable the billing of such complex but efficient energy usage.

Value Creation: Fundamental change of the established value generation logic.

Data Complexity: Heterogeneous data sets from various partners need to be exchanged and shared.

Business Complexity: The implementation of big data applications with breakthrough/disruptive potential is the most challenging business approach. Usually it relies on the interplay of various partners that have managed to establish an effective collaboration. New data sources are aggregated and used in order to develop new products and services. By addressing a new market (segment), breakthrough applications - as the name indicates - have the potential to revolutionise established market settings. It is likely that new players will emerge that are better suited to provide data-based service than the established player, and that the underlying business processes will change fundamentally.

\section{Data-Driven Business Opportunities}

In general, the concept of business opportunity is very broad, and is used to describe the chance to address a particular market need through the creative combination of resources that allows the delivery of advanced value propositions (Ardichvili et al. 2003). In this way, the definition of promising business opportunities relies on the balancing of - often mainly technical - capabilities on the supply side, with user needs and interests as well as market dynamics shaping the demand side. In addition, studies indicate that most successful entrepreneurs and investors continuously observe the demand side very carefully in order to understand what customers and 
marketplaces want, and never lose track of this information (Spinelli and Adams 2012). The knowledge reflecting the demand side is used to guide the scoping of offerings by combining own innovative technology components with reusable and available assets from others in a way that fosters competitiveness. In addition, the development of business opportunities is described as a continuous process that involves proactive efforts to explore all essential steps of a new business.

Any innovative technology that is not aligned with a concrete application triggering concrete demand is likely to fail. This is also true for big data solutions. Hence, the successful implementation of big data solutions requires transparency concerning the following four questions:

1. Who is using the new solution? (target user)

2. Who is providing the data? (data)

3. Who is paying for the solution? (revenue model)

4. Who needs to adopt the solution to bring it to the market? (ecosystem)

For instance, the implementation of health data analytics solutions for improved treatment effectiveness by aggregating longitudinal health data requires high investments and resources to collect and store patient data, for instance by means of a dedicated Electronic Health Record (EHR) solution (data). Although it seems to be quite obvious how the involved stakeholders, such as patients, payors, government or healthcare providers, could benefit from aggregated data sets (target user), it remains unclear whether they would be willing to pay (revenue model) or adopt such an implementation (ecosystem). In addition, as the sharing of personal health data is subject to high security and privacy constraints, one needs to clarify under which conditions the healthcare provider who produced and thus owns the data can and is willing to share the patient data (data).

The aforementioned responsibilities might be distributed across organisational boundaries. If the business approach is mainly targeting the optimisation and improvement of existing offerings, the identification of data-driven business opportunities is often within the scope of established partnerships and capabilities. However, if the business approach is aiming at a collaborative setting within new market and business domains, the scoping of business opportunities easily becomes a challenging task with many unknown variables that often cannot even be influenced by the organisation, as elaborated below:

- Big data applications often rely on high investments to ensure data availability: The collection and maintenance of comprehensive and high-quality data sets not only requires high investments but often takes some years until the data sets are comprehensive enough to produce good analytical results. For instance, in the medical domain, one would need to collect large-scale, high-quality and longitudinal data in order to gain reliable insight about the progress of diseases over time. As such high and long-term-based investments often can't be covered by one single party, the conjoint engagement of multiple stakeholders might be required. 
- Collaboration with partners with diverging interests: As the impact of big data solutions increases and more data sources can be aggregated, an effective collaboration of multiple stakeholders with potentially diverging or even opposing interests needs to be established. In addition, the stakeholder's individual interests and constraints might even change over time. In the German electricity market liberalisation, a new market role of metering service providers was created in 2010. They are responsible for harvesting the energy usage data and could foster whole new branches of business. In addition, a range of stakeholders will require data on energy usage: retailers, network operators and new players that offer energy-related services like demand response. However, in order to establish the basis for an effective collaboration, the interests of the various stakeholders need to be reflected when developing the business case. Especially, the ambiguous regulatory framework on the rights and responsibilities of smart data usage prevents the existing and potential new players in utilities business to take on the new role of metering service provider.

- Technological capabilities as well as its cost are a moving target: Not only technological capabilities but also their cost factors are changing fast. Computing power and memory space per unit costs are still progressing exponentially according to Moore's Law. Additionally, an innovative and cost-effective form of information processing that is the main characteristic of all big data technologies decreases the cost and update cycles of technologies considerably. Thus, the cost factor of technological investments needs to be accounted for in the overall calculation.

Having explained why the development of data-driven business opportunities is very challenging, we need to emphasise that the lack of a business case should not hinder investments in big data projects. Instead, organisations should actively engage the emerging data ecosystems that will allow them to gain access to promising user groups and target customers, data assets and technologies, and stakeholders.

\section{Leveraging the Data Ecosystems}

As the impact of most big data applications increases exponentially, more data (scale) from different data sources (scope) can be integrated and analysed. In addition, the deployment of big data applications in industrial and public environments relies on incorporating the domain knowledge of underlying processes, as well as the alignment of many other horizontal technologies (e.g. cybersecurity, HPC, Internet of things, communication) and established systems. Therefore, the implementation of big data applications requires the collaboration of multiple - often competing - stakeholders on various levels: (a) for sharing the data assets; (b) for sharing technology, skills and knowledge with partners and stakeholders and (c) for establishing value networks generating new business. 
Thus, the majority of big data business will take part in ecosystems. Successful ecosystems can help whole economic sectors as well as single players to prosper and develop. However, the governance of ecosystems relies on a balanced give and take. Looking at the various types of data, assets and actors in the data ecosystem will help to illustrate the underlying incentives and roles. The successful governance of big data ecosystems needs to reflect the interests and strategies of all players involved. We can distinguish ecosystems on three different levels.

\subsection{Data-Sharing Ecosystem}

The impact of big data applications increases if the multiple data sources from the various stakeholders of an industrial sector are integrated. For instance, in healthcare, by aggregating the administrative data and financial data with clinical data, it becomes possible to gain insights about the outcome of treatment bundles in terms of resource utilisation. Thus, cooperative settings for the sharing of data are needed. In order to establish sustainable data-sharing ecosystems, it is important to understand:

- Which data source(s) each actor can potentially provide

- What his or her sharing incentives are

- Which requirements (e.g. privacy standards, "opt out" ability, business models) need to be in place in order to enable/foster the sharing of data

For those who are providing data, a mechanism must be developed to ensure transparency and control of data usage, as well as some added value that is enough motivation to provide the data. Individuals might want to receive improved offerings and services with added value or better prices. Companies are interested in data to improve their knowledge about the consumer in order to customise their offerings, increase customer binding or optimise their pricing strategy.

\subsection{Data Innovation Ecosystems}

The data innovation ecosystem is complex and diverse. It contains multiple types of stakeholders, and, to be effective, there needs to be alignment and collaboration between them. It is the "agora" for the sharing of assets, technology, skills and knowledge. It provides scale to achieve consensus and critical mass around the development of AI value through innovation that no single partner alone could achieve (Zillner et al. 2020). It expresses the collaborative purpose that binds organisations and individuals together in achieving successful deployment of AI. The ecosystem is typically composed of the following roles: 
- End User: Person or organisation from different sectors (private and public) that leverages AI technology and services to their advantage

- Application Provider: An organisation that uses AI technology for developing a vertical AI application (e.g. to be offered as AI service)

- User: A person who either knowingly or unknowingly uses or is impacted by a system product or service that uses AI

- Data Supplier: Person or any organisation (public or private) that creates, collects, aggregates and transforms data from both public and private sources

- Technology Creator: Typically, an organisation (of any size) that creates tools, platforms, services, hardware and technical knowledge

- Broker: An organisation that connects the supply and demand for AI assets (such as skills, data, algorithms and infrastructures) needed for developing AI applications by providing a channel for exchanging AI assets

- Innovator/Entrepreneur: Drives the development of innovative AI technology, products and services

- Researcher/Academic: Researches and investigates new algorithms, hardware, technologies, methodologies and business models; provides skills and training in AI and assesses the societal aspects of its impact

- Regulator: Assesses AI systems in compliance with regulation, privacy and legal norms

- Standardisation Body: Defines technology standards (consensus-based, de facto and formalised) to promote the global adoption of AI technology

- Investor/Venture Capitalist: Provides resources and services to develop the commercial potential of the ecosystem

- Citizen: A person who will or will not develop trust in AI technologies

An effective data innovation ecosystem facilitates the cross-fertilisation and exchange between stakeholders that leads to new data-powered value chains that can improve business and society and deliver benefits to citizens.

\subsection{Value Networks in a Business Ecosystem}

Business ecosystems can be defined as "a dynamic structure which consists of an interconnected population of organizations. These organizations can be small firms, large corporations, universities, research centres, public sector organizations, and other parties which influence the system" (Brynjolfsson and McAfee 2012; Peltoniemi and Vuori 2004). They allow organisations to access and exchange many different aspects of value, resources and benefits.

The data economy relies on value networks. In the data-driven economy, value streams are no longer bi-directional but involve several players exchanging different types of value. The party who is benefiting from a value-added service no longer needs to be the one who is paying for the service. Such value networks already exist in the Internet environment. 
Most of the established players providing database solutions, such as Google, eBay, YouTube, Facebook and iTunes, are building up a growing user community by offering free services, which allows them to increase their income as each advertising company is paying a fee per click or user.

\section{Data-Driven Innovation Framework and Success Stories}

The economics of data has a strong impact on the development of data-driven business opportunities. For instance, data can be consumed an unlimited number of times without losing its value, and it can be reused as input for the production of different goods and services. However, its value still depends on complementary assets related to the capability to extract information out of the data (OECD 2015). Given the mentioned economic properties, disruptions through data are becoming more likely. In particular, due to network effects as well as the simplicity of how a variety of offerings with different value/price tags can be brought to the market, the success of data-driven innovation requires continuous alignment between the needs on the demand side and the opportunities on the supply side.

So how can data-driven business opportunities be screened? The data economy in general is a highly dynamic market. This is supported by the rapid growth of the European data markets, as well as recent technical breakthroughs that were made possible by the availability of large volumes of data, such as the Jeopardy demo by IBM Watson or Google Now or Siri. In addition, experts continue to highlight the wide range of commercial opportunities that can be realised by using the technologies available today.

Entrepreneurs bring new offerings to the market and should continuously scan the market's offerings to identify promising available technology components that can be reused to speed up the development time of their innovation. At the same time, although they are confronted with the highly dynamic market, they have to constantly investigate their own unique selling point and the competitiveness of their offering. To stay competitive in this fast-moving market, entrepreneurs need to continuously reassess what is part of their core offering and in which areas they are partnering with others.

The high-growth scenario ${ }^{3}$ in the comprehensive European data market study (European Commission \& Open Evidence 2017) is based on supply-demand dynamics that shift from technology push to demand pull. In other words, any means that provides guidance in match-making between market needs on the demand side and technical capabilities on the supply side helps to stimulate the development of datadriven innovation and in consequence the growth of the European data market.

To summarise, data-driven business opportunities should be described with a clear scope of offering per market segment (supply side) and reflect the ecosystem

\footnotetext{
${ }^{3}$ Which estimated 4\% of GDP growth between 2016 and 2020.
} 


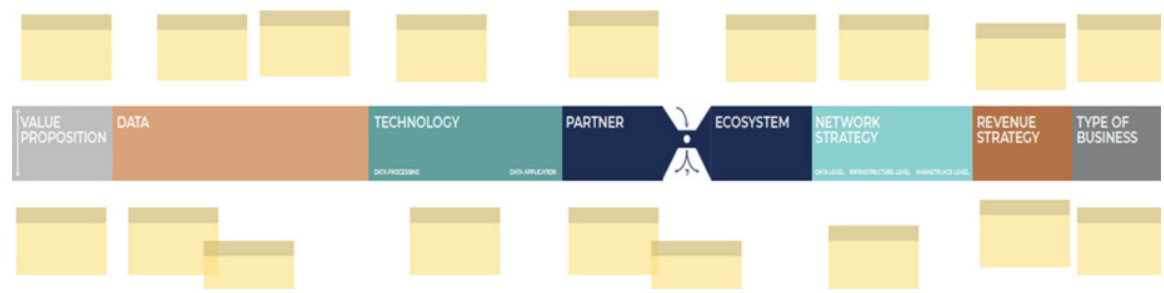

Fig. 2 DDI Canvas with eight dimensions guiding the exploration of the relevant aspects of DDI

dynamics and benefits of network effects (demand side). In the next section, we present a high-level overview of the data-driven innovation framework which guides innovators to systematically explore and analyse the supply and demand sides of data-driven business opportunities by incorporating the particularities of data.

\subsection{The Data-Driven Innovation Framework}

The data-driven innovation (DDI) framework addresses the challenges of identifying and exploring data-driven innovation in an efficient manner. It guides entrepreneurs in scoping promising data-driven business opportunities by reflecting the dynamics of supply and demand through investigating the co-evolution and interactions between the scope of the offering (supply) and the context of the market (demand) in a systematic manner.

The DDI framework is based on a conceptual model in the form of an ontology with a set of categories and concepts describing all relevant aspects of data-driven business opportunities. Its categories are divided into supply side and demand side aspects. On the supply side the focus is on the development of new offerings. For a clearly defined value proposition, this includes the identification of and access to required data sources, as well as the analysis of underlying technologies. On the demand side the focus is on the dynamics of the addressed markets and associated ecosystems. The analysis includes the development of a revenue strategy, a way forward in how to harness network effects as well as an understanding of the type of business. As data-driven innovations are never done in isolation, the identification and analysis of potential development partners as well as partners in the ecosystem help to align/balance the supply and demand aspects in such a way that their competitive nature will stand out. Figure 2 illustrates the DDI Canvas that covers eight central dimensions to be explored when scoping data-driven innovation.

The DDI framework was developed and tested in the context of the Horizon 2020 BDVe project ${ }^{4}$ and is backed by empirical data and scientific research encompassing

\footnotetext{
${ }^{4}$ Zillner. S. D 2.7 Annual Report on Opportunities (BDVe Deliverable), March 2020 Zillner. S. D 2.6 Annual Report on Opportunities (BDVe Deliverable), April 2019 and Zillner. S. et al.: D 2.5 Annual Report on Opportunities (BDVe Deliverable), March 2018.
} 
a quantitative and representative study of more than 90 data-driven business opportunities. The results of the research study guided the fine-tuning and updating of the DDI framework and helped to identify success patterns of a successful data-driven innovation.

Currently the DDI framework is used to run workshops for projects of the BDV Public-Private Partnership, data-driven start-ups and SMEs, and with corporates. It consists of:

- DDI Canvas guiding users in exploring all relevant dimensions on the supply and demand sides of a data-driven innovation in a systematic manner.

- DDI Navigator and methods that support users in exploring each dimension at the required level of detail by investigating the aspects mentioned by guiding questions as well as by applying complementary methods.

- Specific DDI Tools will help the user to work through each of the eight DDI dimensions, producing a conclusive set of results that will guide a companyspecific setup of new, data-based products and services.

More details can be found in Chapter "Big Data Value Creation by Example" of this book, at https://ddi-canvas.com/ or in Zillner and Marangoni (2020), Zillner (2019) or Zillner et al. (2018).

\subsection{Examples of Success Stories}

In the following section, we provide some examples of success stories of the aforementioned research study of data-driven start-ups to give the reader an impression of how clearly and precisely their supply and demand sides can be pitched.

Artomatix is a Dublin-based software company founded in 2014 that uses artificial intelligence to create realistic 3D art creations.

Artomatix's users are artists and developers of the video gaming industry that can benefit from a service that supports the realistic 3D art generation of textures and texturing. Previously, this tedious task was done manually but with the suite of tools provided by Artomatix, artists can now do the same task ten times faster.

The technology is based on computer graphics, Deep Learning and computer vision. It uses generative neuronal networks to "imagine" new details of a texture in a way a human would, i.e. it recognises objects in a video and can add texture and features automatically by relying on the "learned" knowledge that should be there.

The data used for training and developing the algorithm is video and image data. The software can be integrated with Photoshop and leading gaming engines like Unity and Unreal.

The company uses three different subscription models (Indie (revenue $<\$ 100$ $\mathrm{K} /$ year), Professional (revenue $<\$ 1 \mathrm{M} /$ year) and Enterprise (revenue $>\$ 1 \mathrm{M} /$ year)). Enterprises can license Artomatix's technology and build it into their existing process for an annual fee. The technology is offered as a data-driven service. 
There are no network effects that need to be reflected. A short summary is provided in Fig. 3.

\subsubsection{Selectionnist}

Selectionnist is a France-based company founded in 2014 offering image recognition technology with the goal of connecting readers of print journals with the world's largest brands through an application or a chatbot. They aim to bridge the gap between offline content and online experience by offering an advanced matchmaking service to connect consumer and brands.

They address two different customer groups with different value propositions:

- Value proposition for consumer: they locate and potentially purchase a product they spot in a magazine just by snapping a picture of it.

- Value proposition for brands: brands can see in real time how readers interact with their editorial and advertising in print magazines.

Selectionnist's match-making algorithm is based on image recognition technology that continuously improves the images of brands' products in their databases (more brands) and the user request they receive. Thus, their offering is based on network effects on data level. The service is conceptualised as marketplace based on commission fee and with network effects on marketplace level. A short summary of the above explanations is provided in Fig. 4.

\subsubsection{Arable}

Arable is a US-based company founded in 2013 offering agriculture businesses a global solution for managing weather and crop health risks, delivering real-time, actionable insights from the field.

The target users are growers, advisors and businesses who aim to play a proactive role in the quality and longevity of their operations.

The agricultural business intelligence solution is based on in-field measurements allowing the production of real-time continuous visibility and predictive analytics in the areas of crop growth, harvesting time, yield and quality. The solution relies on field-level weather and crop monitoring devices (hardware that is part of the solution) that collect over 40 field-specific data metrics. To enable access to data from anywhere in real time, a cloud-based software platform based on a tiered SaaS offering (different levels of services) is combined with IoT hardware.

Arable sells licences for enterprise software to agribusinesses. As the prediction service improves with more data available, the solution of Arable is based on network effects on a data level. Figure 5 summarises the above-described findings. 


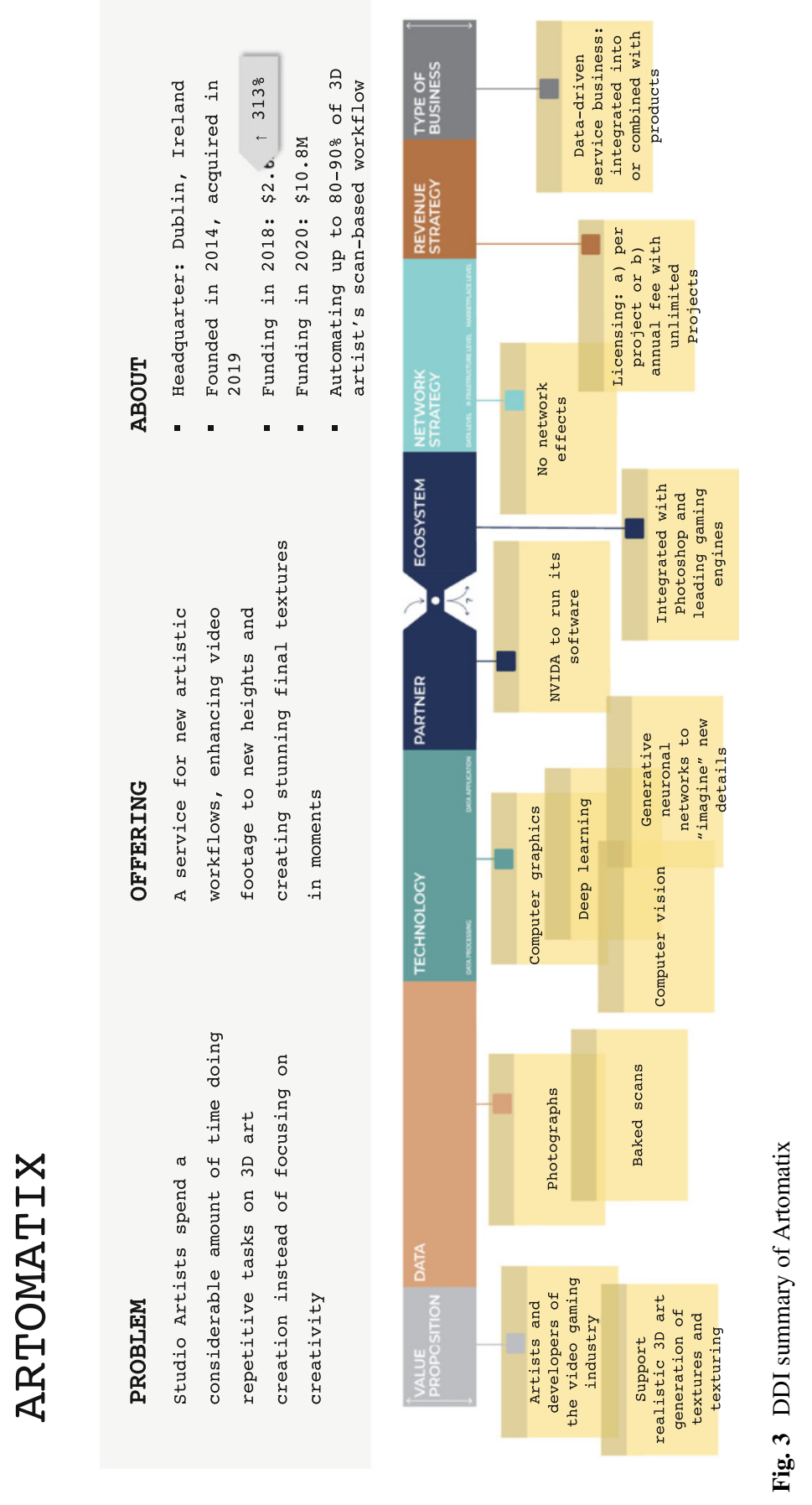




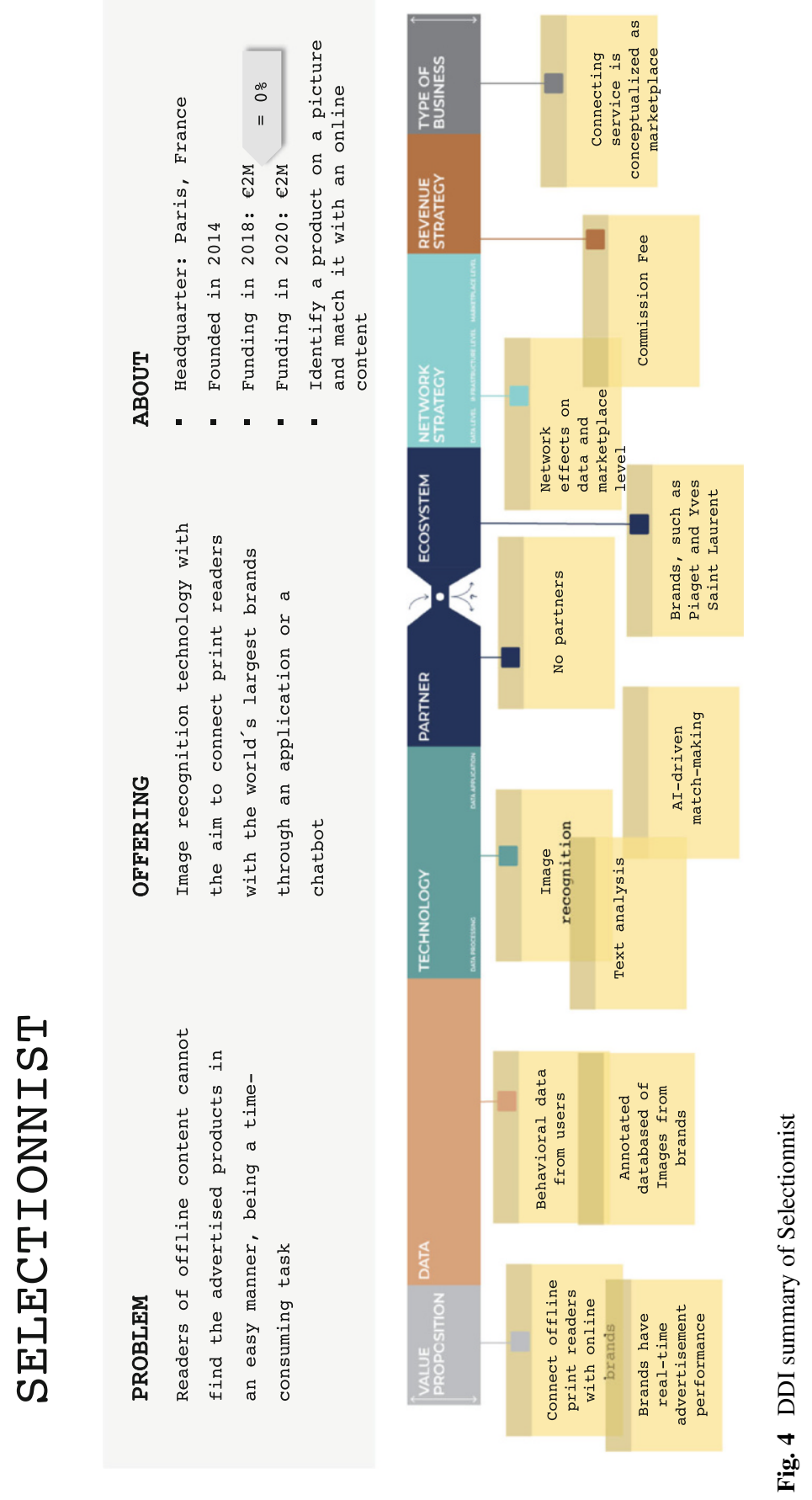




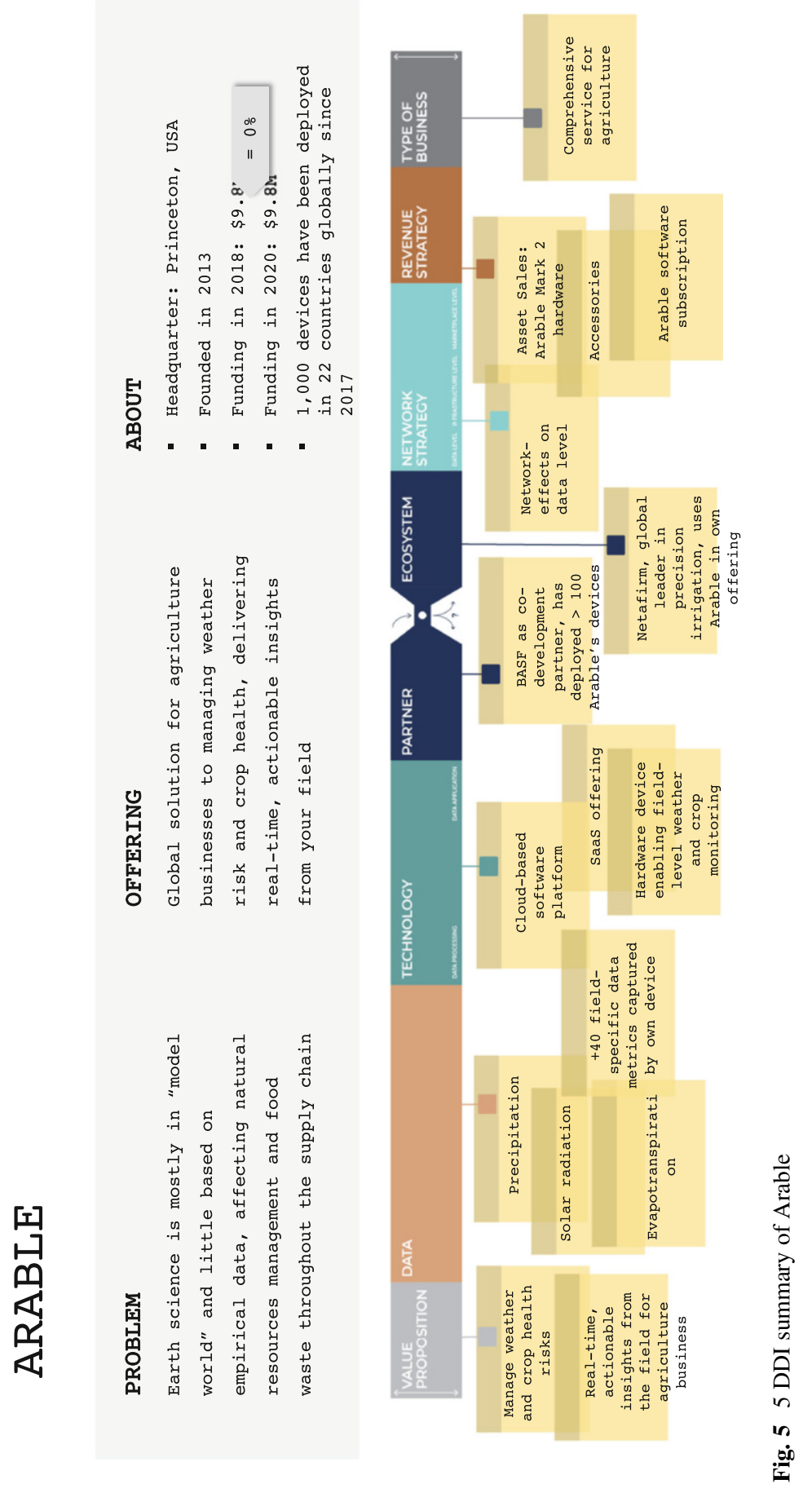




\section{Conclusion}

Big data allows new value to be brought to existing and new businesses. To capture these new types of value, the scoping of data-driven business opportunities needs to incorporate multiple perspectives, ranging from user needs, data availability and technical capabilities to the sustainable establishments of partnerships and ecosystems.

The data-driven innovation framework offers a proven method for all members of the BDV ecosystem to provide guidance in exploring and scoping data-driven business opportunities. The comprehensive content can be used for industrial workshops and educational setups.

\section{References}

Ardichvili, A., Cardozo, R., \& Ray, S. (2003). A theory of entrepreneurial opportunity identification and development. Journal of Business Venturing, 18(1), 105-123. https://doi.org/10.1016/ S0883-9026(01)00068-4

BITKOM. (2013). Management von Big-Data Projekten. Leitfaden des BITKOM AK Big Data.

Brynjolfsson, E., \& McAfee, A. (2012). Winning the race with ever-smarter machines. MIT Sloan Management Review, 53(2), 53-60.

European Commission, (IDC), I. D. C., \& Open Evidence. (2017). Final results of the European Data Market study measuring the size and trends of the EU data economy.

OECD. (2015). Data-driven innovation: Big data for growth and well-being. OECD digital economy papers, p. 456. https://doi.org/10.1787/9789264229358-en

Peltoniemi, M., \& Vuori, E. (2004). Business ecosystem as the new approach to complex adaptive business environments. Proceedings of EBusiness research forum.

Spinelli, S., \& Adams, R. (2012). New venture creation: Entrepreneurship for the 21st century.

Zillner, S. (2019). D2.6. Annual report in opportunities, BDVe deliverable 2.6.

Zillner, S., \& Marangoni, I. (2020). D2.7 Annual report on opportunities; BDVe deliverable 2.7.

Zillner, S., Curry, E., Metzger, A., Auer, S., \& Seidl, R. (Eds.). (2017). European big data value strategic research \& innovation agenda. Retrieved from big data value association website: www.bdva.eu

Zillner, S., Timan, T., \& Kotterink, B. (2018). D2.5 Annual report in opportunities.

Zillner, S., Bisset, D., Milano, M., Curry, E., Hahn, T., Lafrenz, R., et al. (2020). Strategic research, innovation and deployment agenda - AI, data and robotics partnership. Third Release (3rd). Brussels: BDVA, euRobotics, ELLIS, EurAI and CLAIRE. 
Open Access This chapter is licensed under the terms of the Creative Commons Attribution 4.0 International License (http://creativecommons.org/licenses/by/4.0/), which permits use, sharing, adaptation, distribution and reproduction in any medium or format, as long as you give appropriate credit to the original author(s) and the source, provide a link to the Creative Commons licence and indicate if changes were made.

The images or other third party material in this chapter are included in the chapter's Creative Commons licence, unless indicated otherwise in a credit line to the material. If material is not included in the chapter's Creative Commons licence and your intended use is not permitted by statutory regulation or exceeds the permitted use, you will need to obtain permission directly from the copyright holder. 